\title{
Conical refraction mode of an optical resonator
}

\author{
YU. V. LoIko,,$^{1,2 *}$ A. TURPIN, ${ }^{3}$ G. S. SOKOLOVSKII, ${ }^{4,5}$ AND E. U. RAFAILOV ${ }^{1}$ \\ ${ }^{1}$ Aston Institute of Photonic Technologies, School of Engineering \& Applied Science, Aston University, Birmingham, B4 7ET, UK \\ ${ }^{2}$ Social Science Research Institute, Duke University, Durham, NC 27708, USA \\ ${ }^{3}$ University of Glasgow, School of Physics \& Astronomy, Kelvin Building, Glasgow G12 8QQ, Lanark, Scotland \\ ${ }^{4}$ Ioffe Institute, 26 Polytechnicheskaya str., St Petersburg, 194021, Russia \\ ${ }^{5}$ Saint-Petersburg State Electrotechnical University (LETI), 5 Prof. Popova str., St. Petersburg, 197022, Russia \\ *Corresponding author: yury.loika@duke.edu
}

Received XX Month XXXX; revised XX Month, XXXX; accepted XX Month XXXX; posted XX Month XXXX (Doc. ID XXXXX); published XX Month XXXX

\begin{abstract}
The fundamental mode of a conical refraction resonator, i.e. an optical cavity where light experiences conical refraction (CR) from a biaxial crystal, is experimentally demonstrated in the plano-concave cavity configuration. We have discovered that the fundamental CR mode is characterized by the polarization and intensity structures of CR beams between the plane mirror and CR crystal and it resembles the fundamental Gaussian mode with homogeneous polarization between the crystal and concave mirror. We theoretically explained this fundamental CR mode using the dual cone model and symmetry of the CR phenomenon and confirmed this explanation by numerical simulations.
\end{abstract}

\section{(C) 2019 Optical Society of America}

OCIS codes: (140.4780) Optical resonators; (120.2230) Fabry-Perot, (260.5430) Polarization; (260.1440) Birefringence; (260.1180) Crystal optics; (120.5710) Refraction.

http://dx.doi.org/10.1364/OL.99.099999

Optical resonators/cavities are indispensable parts of lasers [1,2] and some types of interferometers [3]. Different types of resonator modes are distinguished by longitudinal and transverse distribution of light intensity and light polarization [1, 2, 4 - 8]. There are two main types of polarization modes [1,2]: modes with (i) homogeneous and (ii) inhomogeneous states of polarization (SOP). Linearly polarized (LP) modes are well known examples of the former type [1, 2]. Azimuthally and radially polarized modes are examples of the latter type $[1,2,9,10]$.

Intracavity birefringent crystals can be used to select resonator modes with either homogeneous or inhomogeneous SOP $[2,9,10]$. LP modes are eigenmodes of such resonators when crystal optic axis and resonator axis are misaligned [1,2]. Azimuthally and radially polarized modes have been observed when the optic axis of uniaxial crystal and resonator optical axis are aligned $[9,10]$.

Azimuthally and radially polarized modes [9-13] are examples of cylindrical vector (CV) beams $[14,15]$. They possess cylindrical axial symmetry of SOP, which is a consequence of axial symmetry of resonator optical anisotropy. These unique properties of CV beams can lead to new interesting applications [15], for instance, in tight focusing [16,17], high-resolution molecular imaging [18] and material processing $[12,13]$.

Recently, there has been an increased interest in the study of light beams obtained under the conical refraction (CR) phenomenon [19-22], i.e. when light propagates along one of optic axes of a biaxial crystal (BC) [23-37]. These CR beams lack axial symmetry. They represent another kind of inhomogeneous SOP where any opposite points are orthogonally linearly polarized, in contrast to CV beams which have opposite points with the same SOP. Applications of CR beams for free-space optical communication [27], particle trapping and manipulation [28], polarization metrology [30], Bose-Einstein condensates [35], and highharmonic generation [36] have already been demonstrated, see also recent review [37]. Recently, beams with CR output patterns from lasers with conical refraction of light inside their cavities have been demonstrated [38-44]. The understanding of the formation of such CR-like modes in lasers and in optical resonators is lagging. In this article, the fundamental $C R$ mode is experimentally demonstrated in the plano-concave resonator by exploring the CR phenomenon in an intracavity biaxial crystal for mode selection. This fundamental CR mode appears when one of the optic axes of the crystal and resonator axis are aligned, see Fig. 1. We call this configuration a CR resonator because the observed CR mode between the crystal and plain mirror resembles the polarization and intensity distribution of a CR beam, however, it resembles an ordinary fundamental Gaussian beam with homogeneous SOP between the crystal and curved mirror. Moreover, we demonstrate how a light beam is transformed during and restored after a singleround trip in the CR resonator. For these purposes, we explored cascaded CR in reflection geometry when a light beam passes along the optic axis of a biaxial crystal, reflects from a plane mirror and passes backward through the same crystal. The results reported in this article are crucial for the development of new applications of CR in the field of lasers, optical resonators and optical interferometry because they provide us with a deeper understanding of the formation of CR modes in optical cavities. 


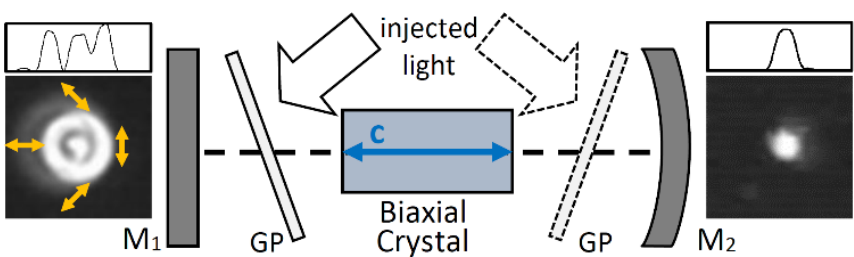

Fig. 1. (color online) Fundamental CR mode. Experimental setup (center). Characteristic CR intensity and polarization pattern observed at plane mirror $\mathrm{M}_{1}$ (left) and fundamental Gaussian intensity profile with homogeneous SOP at curved mirror $\mathrm{M}_{2}$ (right) when circularly polarized light from an external laser is injected through a glass plate (GP, solid line) close to the plane mirror $\mathrm{M}_{1}$. Optic axis $\mathbf{c}$ of intracavity biaxial crystal is aligned along the resonator axis (dashed line). Another optic axis of the crystal is in the plane perpendicular to the figure. Similar patterns are observed when light is injected between the crystal and curved mirror $\mathrm{M}_{2}$ (GP, dotted lines). Double arrows show polarization distribution. The insets above the beam images show intensity distributions through the centers of corresponding patterns.

Conical Refraction Resonators are resonators where propagating light experiences the CR phenomenon within the cavity. The simplest configuration of a CR resonator is shown in Fig. 1. It is comprised of a biaxial crystal with one of its optic axes (c) aligned along the optical axis of a plano-concave or hemi-spherical resonator formed by plane $\mathrm{M}_{1}$ and concave $\mathrm{M}_{2}$ mirrors.

In our setup (Fig. 1) we used the $\left.\mathrm{Nd}: \mathrm{KGd}_{(} \mathrm{WO}_{4}\right)_{2}(\mathrm{Nd}: \mathrm{KGW}$ ) biaxial crystal (3 at.\% doping) (22 mm long with cross-section 3x4 mm², CR ring radius of $\mathrm{R}_{0}=0.37 \mathrm{~mm}$ ) placed between plane $\mathrm{M}_{1}$ and concave $\mathrm{M}_{2}$ (radius of curvature ROC $=200 \mathrm{~mm}$ ) mirrors. The distance between mirrors was $125 \mathrm{~mm}$, which determined the Gaussian mode with a beam waist radius of $\mathrm{w}_{0}=0.18 \mathrm{~mm}$ in a nonCR resonator. Such a beam waist defines the characteristic parameter $\rho_{0}=R_{0} / w_{0}=2.06$ of the CR phenomenon $[22-25,29,33$, 34]. A glass plate (GP) inclined to the resonator axis was used for coupling an external laser light into the cavity. As injected light, we used an output beam from an electrically pumped vertical external cavity surface emitting semiconductor laser (EP VECSEL) $(\lambda=$ $980 \mathrm{~nm}, \mathrm{M}^{2} \sim 10$ ) magnified to a diameter of $12 \mathrm{~mm}$ and transformed to circular polarization.

Transverse patterns of the observed CR resonator mode at the plane $\mathrm{M}_{1}$ and concave $\mathrm{M}_{2}$ mirrors are presented in Fig.1, see left and right inset respectively, for a configuration when external light was injected between the crystal and the plane mirror $\mathrm{M}_{1}$ (Fig.1, GP solid line). This configuration allows one to distinguish whether a CR mode or a single pass CR phenomenon is observed. In the latter case, a CR ring pattern [26] would appear at the concave mirror $\mathrm{M}_{2}$, which did not occur in our case (see insets in Fig. 1). We observed similar transverse patterns as in Fig.1 when injected light was coupled through a GP (dotted line in Fig.1) placed between the crystal and concave mirror $\mathrm{M}_{2}$.

The observed resonator mode can be called fundamental CR mode because it possesses two remarkable characteristics. (i) Its transverse pattern at the plane mirror (Fig.1, left inset) resembles the intensity and polarization structure of the CR pattern observed in CR experiments [26-29]. In particular, the observed mode has a bright ring structure with inhomogeneous SOP distribution at the plane mirror as a CR beam would have at the focal (Lloyd) plane, i.e. any two opposite points on the ring have linear orthogonal polarizations and the direction of polarization is continuously varied along the ring. (ii) The observed mode at concave mirror has a fundamental Gaussian intensity profile (with goodness of fit $\mathrm{R}^{2}=98.6 \%$ ) with homogeneous SOP (Fig.1, right inset).

In order to ensure that the observed CR mode is not a high-order mode of an ordinary (non-CR) resonator, we have captured images at different planes far from the plane mirror, see Figs. 2(a) to 2(d). At the plane mirror, the fundamental CR mode, Fig. 2(a), has characteristic patterns of a CR beam with a central spot and bright ring, which would also be observed under the CR phenomenon for the characteristic parameter $\rho_{0}=2.06$ (see also Ref. [29]). Fig. 2 clearly demonstrates two CR cones. Their cross-section looks like concentric rings and their diameters increase as the distance from the plane mirror increases, i.e. from Fig. 2(b) to Fig. 2(d). These cones are characteristics of CR beams [31,32]. The central bright spot in Fig.2(b) is another remarkable manifestation of CR beams. It corresponds to the Raman spot (vertex of one of two CR cones) [21] usually observed in CRexperiments [26]. One needs to mention that the diameter of one of the CR cones, which forms the Raman spot, decreases between the plane mirror (outer ring in Fig.2(a)) and the Raman spot (Fig.2(b)).
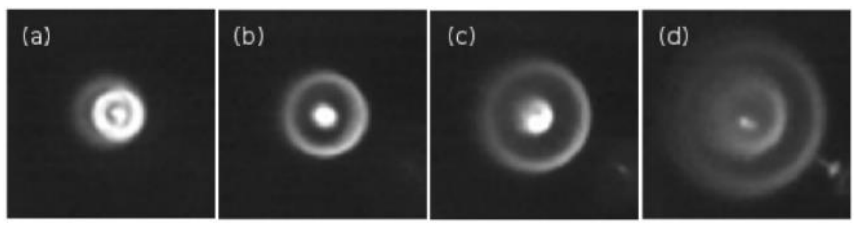

Fig. 2. The light beam propagating out of the plane mirror (a-d) for fundamental CR mode, which was excited in the CR resonator shown in Fig.1. Two CR cones expand from the plane mirror, figures (a) through (d). The central spot in figure (b) corresponds to the vertex (Raman spot) of one of the cones. The distance between the planes of consecutive images was $12 \mathrm{~cm}$. Additional small spots in figures (c-d) correspond to the reflection of injected light from the crystal's edges.

Conical refraction within single round trip in CR resonator. In order to gain insight into the mechanisms of mode formation inside CR resonators, we have performed a set of experiments on CR in reflection geometry, see Fig. 3. In this configuration a light beam passes through a biaxial crystal, reflects from a mirror behind the crystal and passes back through the same crystal.

The scheme of our experimental setup is shown in Fig. 3. Collimated linearly polarized light from EP VECSEL described above is split by a non-polarizing beam splitter (NP BS, 50/50) to form two identical beams, a reflected reference beam and a transmitted signal beam. The latter one is focused by lens $\mathrm{L}$ into a biaxial crystal (BC) along one of its optic axes. This beam is refracted conically by the crystal. A CR crescent (ring) structure is formed at the focal (Lloyd) plane, see Fig. 3(b). We used a plano-convex focusing lens with a focal distance of $f=75 \mathrm{~mm}$ and a $l=6 \mathrm{~mm}$ long Nd:KGW crystal ( 3 at.\% doping) cut perpendicular to one of its optic axes (misalignment within $1.5 \mathrm{mrad}$ ). The conicity parameter of the Nd:KGW crystal is equal to $\alpha=17 \mathrm{mrad}$, which provides the $\mathrm{CR}$ ring with a radius of $\mathrm{R}_{0}$ $=l \cdot \alpha=0.10 \mathrm{~mm}$ at the focal plane [22 - 26], see Fig. 3(b).

After it has been reflected from the plane mirror $\mathrm{M}_{1}$, the signal beam propagates in the opposite direction through the same system made up of crystal BC and lens L. A part of the backward beam is redirected by the NP BS and registered by a CCD camera. 


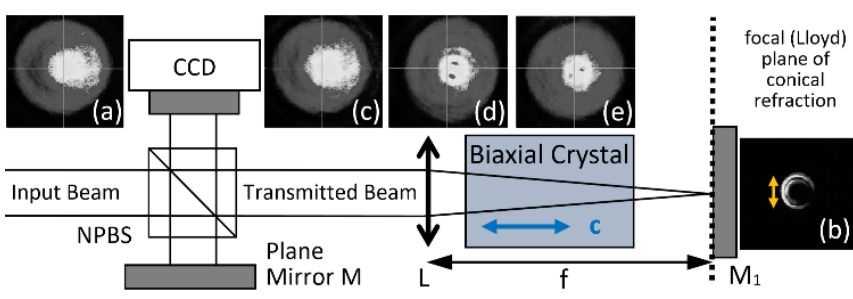

Fig. 3. (color online) Conical refraction in reflection geometry. Nonpolarizing beam splitter (NPBS) splits an input laser beam into two identical beams: reflected and transmitted beams. The former one is registered by a CCD camera as a reference beam after being reflected from a plane mirror $\mathrm{M}$. The transmitted signal beam propagates along an optic axis through a biaxial crystal in the forward and backward directions. Lens $\mathrm{L}$ is used for focusing the forward beam and for collimating the backward beam. Reconstruction of light beam refracted conically (a-e). The input light beam from EP VECSEL (a), see parameters in the text, its $\mathrm{CR}$ crescent pattern at the plane mirror $\mathrm{M}_{1}$ (b) and reconstruction in the opposite direction (c) when the plane mirror surface coincides with the focal (Lloyd) plane (dotted line). The leftmost point of the CR crescent in figure (b) has the same vertical linear polarization (arrow) as the input beam. Any two opposite points of the $\mathrm{CR}$ crescent (ring) are linearly orthogonally polarized. The input beam is not reconstructed, i.e. backward beam distortion is observed, when the surface of the plane mirror $\mathrm{M}_{1}$ is angularly misaligned, for instance, in horizontal (d) or in vertical (e) direction.

The same CCD camera is served to measure the profile of the reference beam reflected from the plane mirror M, see Fig. 3(a).

When a plane mirror is used as the reflector $M_{1}$ and its surface coincides exactly with the focal (Lloyd) plane, the backward beam passes along the crystal optic axis and undergoes CR. This CR transformation has the exact inverse effect compared to the CR effect in the forward direction and, therefore, the input beam is reconstructed after propagation through the crystal $\mathrm{BC}$ and lens $\mathrm{L}$ in the backward direction, see Fig. 3(c). Such configuration of the CR phenomenon in reflection geometry is equivalent to previously described cascaded CR with two identical and oppositely oriented biaxial crystals [27]. When the surface of the reflecting plane mirror $\mathrm{M}_{1}$ is angularly misaligned from the focal (Lloyd) plane, the backward beam does not propagate along the crystal optic axis, and birefringence of the backward beam can't compensate the CR transformation that the forward beam undergoes, see Figs. 3(d) and $3(\mathrm{e})$. The input beam is not restored when the plane mirror is moved from the focal (Lloyd) plane in either direction.

Dual-cone model of CR resonator modes: Experimental results reported above provide a guide for theoretical modelling and an explanation to mode formation in CR resonators. More intuitive description can be envisaged by utilizing dual-cone model of CR [31, 32]. Let's consider an arbitrary set of waves that form resonator mode and propagate from a concave mirror $\mathrm{M}_{2}$ and undergo $\mathrm{CR}$ in an intracavity biaxial crystal, see Fig. 4 . According to the dual-cone model, CR inside the crystal splits them into two cones (see $\mathrm{C}_{+}$and C-cones and dotted lines in Fig.4). Behind the crystal these cones or their continuation have vertices at different spatial points along the propagation direction. The cones intersect at the focal (Lloyd) plain, the CR symmetry plain. This means that each CR cone is a mirror image of the other one with respect to its reflection in the focal plane. In CR resonators studied here, this mirror symmetry is used for $\mathrm{CR}$ mode formation by aligning the plane mirror $\mathrm{M}_{1}$ (Fig. 4) with the Lloyd plain of CR produced by the intracavity BC. CR mirror symmetry means that after reflecting the $\mathrm{C}_{+} / \mathrm{C}_{-}$cone, a $\mathrm{C}_{-} / \mathrm{C}_{+}$cone is formed, i.e. it acquires the same polarization distribution and the same wavefront as the $\mathrm{C}_{-} / \mathrm{C}_{+}$cone does. When propagating back in space, each cone undergoes inverse transformation in the crystal. This means that the initial set of waves is restored at the crystal exit and it propagates in the opposite direction, i.e. to concave mirror. Therefore, any field distribution with a wavefront that is compatible with mirror $\mathrm{M}_{2}$ will form a resonator mode. In our case, with spherical concave mirror $\mathrm{M}_{2}$, it is the fundamental Gaussian mode.

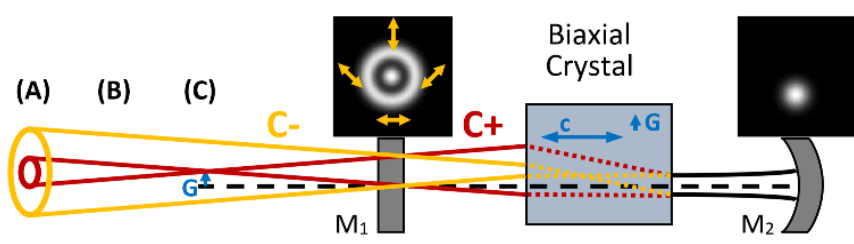

Fig. 4. (color online) Dual cone model of CR resonator modes. $\mathrm{C}_{+}$(red) and $\mathrm{C}_{-}$(yellow) represent two cones of conically refracted light. $\mathrm{C}_{+}$cone has a vertex outside the $\mathrm{CR}$ resonator. Dotted lines represent profiles of $\mathrm{CR}$ cones inside the biaxial crystal. The dashed line represents the resonator axis. Left and right insets show theoretically calculated patterns of the fundamental $\mathrm{CR}$ mode at the plane and concave mirrors with parameters of CR resonator in Fig. 1. (A), (B) and (C) indicate plains where Figs. 2(d), 2(c) and 2(b) were taken. The CR cavity here is the same as in Fig.1 but has been rotated by $90^{\circ}$ around the cavity axis in order to show the lateral shift of the output beam center, given by vector G, with respect to the cavity axis. Vector $\mathbf{G}$ is a characteristic of a biaxial crystal: its orientation depends on the plane containing the two optic axes of the crystal, while its magnitude depends on the length and conicity parameter of the crystal. G indicates a transverse shift of beam center under the CR phenomenon $[22,26]$ when light propagates from concave $\mathrm{M}_{2}$ to plane $\mathrm{M}_{1}$ mirror. It is equal to the vector pointing from the $\mathrm{CR}$ ring point with tangential polarization toward the $\mathrm{CR}$ ring center. Transverse shift for the center of light beam propagating from plane to concave mirror is given by $\mathbf{- G}$.

We performed simulations of the CR resonator mode on the basis of the beam propagation method and theoretical models of CR developed previously [22-25]. We determined parameters of the fundamental gaussian mode at the curved mirror, which provided stationary transverse profiles at both mirrors. Theoretical results are shown in insets in Fig. 4 for CR mode with circular polarization at concave mirror. They corroborate with experimental results reported in Fig. 1. Moreover, our theoretical results, see Fig. 4, demonstrate additional features of $\mathrm{CR}$ resonators and their modes. The center of the CR mode at the plane mirror is laterally shifted with respect to the axis of bulk resonator, i.e. without the crystal. It corroborates with previous observations that any beam that undergoes the CR phenomenon is laterally shifted in the plane of optic axes of a biaxial crystal used for CR [26]. In Fabry-Perot resonators, like the ones considered here, the axis of CR mode between the concave mirror and biaxial crystal coincides with the resonator axis because of axial cylindrical symmetry of the mode formed between them. Waves propagate twice through the crystal during their single round trip. The CR phenomenon inside the crystal breaks axis cylindrical symmetry of waves propagating from the concave to plane mirror and restore this symmetry when they propagate back to the concave mirror. The waves are laterally shifted in the plane of crystal optic axes, but in opposite directions during forward and backward propagation. To clarify, for 
orientation of CR resonators as shown in Fig. 4, the center of light waves propagating from the concave to plane mirror is shifted up by vector G. Its modulus is equal to the radius of the observed CR ring pattern at the focal (Lloyd) plane, i.e. $|\boldsymbol{G}|=R_{0}=\alpha l$. The center of light waves propagating from the plane to concave mirror is shifted down by -G. Consequently, the axial cylindrical symmetry of the resonator mode is restored between the crystal and concave mirror.

In conclusion, we have proposed a new type of optical resonator: the $\mathrm{CR}$ resonator. In CR resonators, conical refraction of light inside the cavity is used for selection of resonator modes. For these purposes we used a biaxial crystal with its optic axis aligned with the resonator axis. We observed fundamental CR mode of simple CR resonator in plano-concave geometry. This mode has (i) inhomogeneous SOP and intensity distribution between the plane mirror and crystal, which resembles the CR beam observed in CR, and (ii) fundamental Gaussian profile between the crystal and concave mirror. The observed mode has a complex structure of phase surface at the plane mirror, which contrasts with the constant phase wavefront of fundamental Gaussian mode with homogeneous SOP. When compared with radially and azimuthally polarized modes, the fundamental CR mode lacks axial cylindrical symmetry in intensity and/or polarization distribution. Moreover, the axis of the output beam is laterally shifted with respect to the resonator axis, which is explained by a lateral shift provided under CR by an intracavity biaxial crystal.

Our results open new unexplored grounds in the fields of optical resonators, optical interferometry, polarimetry, spectroscopy and lasers, and can initiate new studies and discoveries in these fields by exploring optical properties of the CR phenomenon. In particular, our findings suggest that resonator modes with different profiles at the plane mirror, including super-gaussian, single- and double-ring structures, demonstrated in previous CR experiments [37] should be observed in CR resonators and CR lasers. They can be selected by appropriate choice of resonator configuration and biaxial crystals controlled by the parameter $\rho_{0}=\mathrm{R}_{0} / \mathrm{w}_{0}$, which has been partially demonstrated in recent laser experiments with intracavity CR [44]. This makes CR resonators unique instruments to explore the unusual structure and properties of its modes for many new prospective applications. In particular, our results can inspire further theoretical and experimental investigations in the field of CR optical resonators and optical interferometry based on CR. They can lead to the development of new types of optical interferometers with enhanced precision of optical measurements based on strong angular sensitivity of the CR phenomenon. Results reported in this article improve our knowledge about CR resonators and CR lasers [38-43], which can provide better understanding of their eigenmodes and lasing regimes, and, finally, can lead to the development of optimized and efficient schemes of their operation.

Funding. European Union's Seventh Framework Programme (FP7/2007-2013) managed by the Research Executive Agency (http://ec.europa.eu/rea) (314936 FP7-SME-2012). E.U.R. thanks Grant of Ministry of Education and Science 17.1223.2017/PCh for partial support.

Conflict of Interest: The authors declare no conflicts of interest.

\section{References}

1. A. E. Siegman, Lasers (University Science Books, 1986).

2. W. Koechner, Solid-State Laser Engineering (Springer, 2006).
3. P. Hariharan, Optical Interferometry (Elsevier, 2003).

4. N. Hodgson, and H. Weber, Optical Resonators: Fundamentals, Advanced Concepts and Applications (Springer, 1997).

5. A. G. Fox, and T. Li, Proc. IRE 48, 1904 (1960); A. G. Fox, and T. Li, Bell Sys. Tech J. 40, 453 (1961).

6. G. D. Boyd and J. P. Gordon, Bell Sys. Tech J. 40, 489 (1961).

7. G. D. Boyd and H. Kogelnik, Bell Sys. Tech J. 41, 1347 (1962).

8. H. Kogelnik, and T. Li, Applied Optics 10, 1550 (1966).

9. D. Pohl, Appl. Phys. Lett. 20, 266 (1972).

10. J. Wynne, IEEE J. Quantum Electron. QE-10, 125 (1974).

11. Y. Mushiake, K. Matsumura, and N. Nakajima, Proc. IEEE 60, 1107 (1972).

12. V. G. Niziev, and A. V. Nesterov, J. Phys. D 32, 1455 (1999).

13. A. V. Nesterov, and V. G. Niziev, J. Phys. D 33, 1817 (2000).

14. Q. Zhan, Adv. Opt. Photon. 1, 1 (2009).

15. Vectorial Optical Fields: Fundamentals and Applications, ed. by Q. Zhan (World Scientific Publishing Co., 2014)

16. S. Quabis, R. Dorn, M. Eberler, O. Glöckl, and G. Leuchs, Opt. Commun. 179, 1 (2000).

17. R. Dorn, S. Quabis, and G. Leuchs, Phys. Rev. Lett. 91, 233901 (2003).

18. L. Novotny, M. R. Beversluis, K. S. Youngworth, and T. G. Brown, Phys. Rev. Lett. 86, 5251 (2001).

19. W. R. Hamilton, Trans. R. Irish Acad. 17, 1-144 (1837).

20. H. Lloyd, Trans. R. Irish Acad. 17, 145-158 (1837).

21. C. V. Raman, V. S. Rajagopalan, and T. M. K. Nedungadi, Nature 147, 268 (1941).

22. A. M. Belskii, and A. P. Khapalyuk, Opt. Spectrosc. 44, 436 (1978).

23. A. M. Belsky, and M. A. Stepanov, Opt. Commun. 167, 1 (1999).

24. M. V. Berry, J. Opt. A: Pure Appl. Opt. 6, 289 (2004).

25. M. V. Berry, and M. R. Jeffrey, Prog. Opt. 50, 13 (2007).

26. T. K. Kalkandjiev, and M. A. Bursukova, Proc. SPIE 6994, 69940B-1 (2008).

27. A. Turpin, Yu. V. Loiko, T. K. Kalkandjiev, and J. Mompart, Opt. Lett. 37, 4197 (2012).

28. A. Turpin, V. Shvedov, C. Hnatovsky, Yu. V. Loiko, J. Mompart, and W. Krolikowski, Opt. Express 21, 26335 (2013).

29. Yu. V. Loiko, A. Turpin, T. K. Kalkandjiev, E. U. Rafailov, and J. Mompart, Opt. Lett. 38, 4648 (2013).

30. A. Peinado, A. Turpin, A. Lizana, E. Fernandez, J. Mompart, and J. Campos, Opt. Lett. 38, 4100-4103 (2013).

31. G. S. Sokolovskii, D. J. Carnegie, T. K. Kalkandjiev, and E. U. Rafailov, Opt. Express 21, 11125 (2013).

32. A. Turpin, Yu. V. Loiko, T. K. Kalkandjiev, H. Tomizawa, and J. Mompart, Opt. Lett. 40, 1639 (2015).

33. V. Peet, Opt. Express 18, 19566 (2010).

34. A. Turpin, Yu. V. Loiko, A. Peinado, A. Lizana, T. K. Kalkandjiev, J. Campos, and J. Mompart, Opt. Express 23, 5704 (2015).

35. A. Turpin, J. Polo, Y. V. Loiko, J. Küber, F. Schmaltz, T. K. Kalkandjiev, V. Ahufinger, G. Birkl, and J. Mompart, Opt. Express 23, 1638 (2015).

36. A. Turpin, L. Rego, A. Picón, J. San Román, C. Hernández-García, Sci. Rep. 7, 4388-1-4388-10 (2017).

37. A. Turpin, Yu. V. Loiko, T. K. Kalkandjiev, and J. Mompart, Laser Photon. Rev. 10, 750 (2016).

38. K. G. Wilcox, A. Abdolvand, T. K. Kalkandjiev and E. U. Rafailov. Appl. Phys. B 99, 619 (2010)

39. R. Cattoor, I. Manek-Hönninger, D. Rytz, L. Canioni, and M. Eichhorn, Opt. Lett. 39, 6407 (2014).

40. Y. V. Loiko, G. S. Sokolovskii, D. Carnegie, A. Turpin, J. Mompart, and E. Rafailov, Proc. SPIE 8960, 89601Q (2014).

41. A. Brenier, Laser Phys. Lett. 11, 115819 (2014).

42. A. Brenier, Appl. Phys. B 122, 237 (2016).

43. A. Brenier, Laser Phys. 27, 105001 (2017).

44. R. Akbari, C. Howlader, K. A. Fedorova, G. S. Sokolovskii, E. U. Rafailov, and A. Major, Opt. Lett. 44, 642 (2019). 


\section{References}

1. A. E. Siegman, Lasers (University Science Books, 1986).

2. W. Koechner, Solid-State Laser Engineering (Springer, 2006).

3. P. Hariharan, Optical Interferometry (Elsevier, 2003).

4. N. Hodgson, and H. Weber, Optical Resonators: Fundamentals, Advanced Concepts and Applications (Springer, 1997).

5. A. G. Fox, and T. Li, "Resonant modes in an optical maser," Proc. IRE 48, 19041905 (1960); A. G. Fox, and T. Li, "Resonant modes in a maser interferometer," Bell Sys. Tech J. 40, 453-488 (1961).

6. G. D. Boyd and J. P. Gordon, "Confocal multimode resonator for millimeter through optical wavelength masers," Bell Sys. Tech J. 40, 489-508 (1961).

7. G. D. Boyd and H. Kogelnik, "Generalized confocal resonator theory," Bell Sys. Tech J. 41, 1347-1369 (1962).

8. H. Kogelnik, and T. Li, "Laser beams and resonators," Applied Optics 10, 15501567 (1966)

9. D. Pohl, "Operation of a Ruby Laser in the Purely Transverse Electric Mode TE01," Appl. Phys. Lett. 20, 266-267 (1972).

10. J. Wynne, "Generation of the rotationally symmetric $\mathrm{TE}_{01}$ and $\mathrm{TM}_{01}$ modes from a wavelength-tunable laser," IEEE J. Quantum Electron. QE-10, 125 (1974). 11. Y. Mushiake, K. Matsumura, and N. Nakajima, "Generation of Radially Polarized Optical Beam Mode by Laser Oscillation," Proc. IEEE 60, 1107-1109 (1972).

12. V. G. Niziev, and A. V. Nesterov, "Influence of beam polarization on laser cutting efficiency," J. Phys. D 32, 1455-1461 (1999).

13. A. V. Nesterov, and V. G. Niziev, "Laser beams with axially symmetric polarization," J. Phys. D 33, 1817-1822 (2000).

14. Q. Zhan, "Cylindrical vector beams: from mathematical concepts to applications," Adv. Opt. Photon. 1, 1-57 (2009).

15. Vectorial Optical Fields: Fundamentals and Applications, ed. by Q. Zhan (World Scientific Publishing Co., 2014)

16. S. Quabis, R. Dorn, M. Eberler, O. Glöckl, and G. Leuchs, "Focusing light to a tighter spot," Opt. Commun. 179, 1-7 (2000).

17. R. Dorn, S. Quabis, and G. Leuchs, "Sharper focus for a radially polarized light beam," Phys. Rev. Lett. 91, 233901-1-233901-4 (2003).

18. L. Novotny, M. R. Beversluis, K. S. Youngworth, and T. G. Brown, "Longitudinal field modes probed by single molecules," Phys. Rev. Lett. 86, 5251-5254 (2001). 19. W. R. Hamilton, "Third supplement to an essay on the theory of systems of rays," Trans. R. Irish Acad. 17, 1-144 (1837).

20. H. Lloyd, "On the phenomena presented by light in its passage along the axes of biaxial crystals," Trans. R. Irish Acad. 17, 145-158 (1837).

21. C. V. Raman, V. S. Rajagopalan, and T. M. K. Nedungadi, “Conical refraction in naphthalene crystals," Nature 147, 268 (1941).

22. A. M. Belskii and A. P. Khapalyuk, "Internal conical refraction of bounded light beams in biaxial crystals," Opt. Spectrosc. 44, 436-439 (1978).

23. A. M. Belsky and M. A. Stepanov, "Internal conical refraction of coherent light beams," Opt. Commun. 167, 1-5 (1999).

24. M. V. Berry, "Conical diffraction asymptotics: fine structure of Poggendorff rings and axial spike," J. Opt. A: Pure Appl. Opt. 6, 289 (2004).

25. M. V. Berry, and M. R. Jeffrey, "Conical diffraction: Hamilton's diabolical point at the heart of crystal optics," Prog. Opt. 50, 13 (2007).

26. T. K. Kalkandjiev, and M. A. Bursukova, "Conical refraction: an experimental introduction," Proc. SPIE 6994, 69940B-1-69940B-10 (2008).

27. A. Turpin, Yu. V. Loiko, T. K. Kalkandjiev, and J. Mompart, "Free space optical polarization de-multiplexing and multiplexing by means of conical refraction," Opt. Lett. 37, 4197-4199 (2012).

28. A. Turpin, V. Shvedov, C. Hnatovsky, Yu. V. Loiko, J. Mompart, and W. Krolikowski, "Optical vault: A reconfigurable bottle beam based on conical refraction of light," Opt. Express 21, 26335-26340 (2013).

29. Yu. V. Loiko, A. Turpin, T. K. Kalkandjiev, E. U. Rafailov, and J. Mompart, "Generating a three-dimensional dark focus from a single conically refracted light beam," Opt. Lett. 38, 4648-4651 (2013).

30. A. Peinado, A. Turpin, A. Lizana, E. Fernandez, J. Mompart, and J. Campos, "Conical refraction as a tool for polarization metrology," Opt. Lett. 38, 4100-4103 (2013).
31. G. S. Sokolovskii, D. J. Carnegie, T. K. Kalkandjiev, and E. U. Rafailov, "Conical Refraction: New observations and a dual cone model," Opt. Express 21, 1112511131 (2013).

32. A. Turpin, Yu. V. Loiko, T. K. Kalkandjiev, H. Tomizawa, and J. Mompart, "On the dual-cone nature of the conical refraction phenomenon," Opt. Lett. 40, 16391642 (2015).

33. V. Peet, "Improving directivity of laser beams by employing the effect of conical refraction in biaxial crystals," Opt. Express 18, 19566-19573 (2010).

34. A. Turpin, Yu. V. Loiko, A. Peinado, A. Lizana, T. K. Kalkandjiev, J. Campos, and J. Mompart, "Polarization tailored novel vector beams based on conical refraction," Opt. Express 23, 5704-5715 (2015).

35. A. Turpin, J. Polo, Y. V. Loiko, J. Küber, F. Schmaltz, T. K. Kalkandjiev, V. Ahufinger, G. Birkl, and J. Mompart, "Blue-detuned optical ring trap for BoseEinstein condensates based on conical refraction," Opt. Express 23, 1638-1650 (2015).

36. A. Turpin, L. Rego, A. Picón, J. San Román, C. Hernández-García, “Extreme ultraviolet fractional orbital angular momentum beams from high harmonic generation," Sci. Rep. 7, 4388-1-4388-10 (2017).

37. A. Turpin, Yu. V. Loiko, T. K. Kalkandjiev, and J. Mompart, "Conical Refraction: fundamentals and applications," Laser Photon. Rev. 10, 750-771 (2016).

38. K. G. Wilcox, A. Abdolvand, T. K. Kalkandjiev and E. U. Rafailov. "Laser with simultaneous Gaussian and conical refraction outputs," Appl. Phys. B 99, 619 (2010).

39. R. Cattoor, I. Manek-Hönninger, D. Rytz, L. Canioni, and M. Eichhorn, "Laser action along and near the optic axis of a holmium-doped KY(WO4)2 crystal," Opt. Lett. 39, pp.6407-6410 (2014).

40. Y. V. Loiko, G. S. Sokolovskii, D. Carnegie, A. Turpin, J. Mompart, and E. Rafailov, "Laser beams with conical refraction patterns," Proc. SPIE 8960, 896010 (2014).

41. A. Brenier, "Polarization properties of lasing near an optical axis in the biaxial crystal," Laser Phys. Lett. 11, 115819 (2014).

42. A. Brenier, "Lasing with conical diffraction feature in the KGd(WO4)(2):Nd biaxial crystal," Appl. Phys. B 122, 237 (2016).

43. A. Brenier, "Revealing modes from controlling an off-optical axis conical diffraction laser," Laser Phys. 27, 105001 (2017).

44. R. Akbari, C. Howlader, K. A. Fedorova, G. S. Sokolovskii, E. U. Rafailov, and A. Major, "Conical refraction output from a Nd:YVO4 laser with an intracavity conerefringent element," Opt. Lett. 44, 642-645 (2019). 